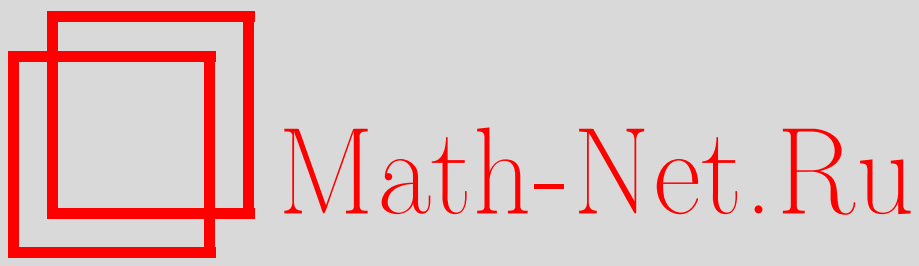

А. В. Чекотило, Оценка погрешностей астрогиросистемы, Вестн. Сам. гос. техн. ун-та. Сер. Физ.-мат. науки, 2005, выпуск 38, 175-177

DOI: https://doi.org/10.14498/vsgtu400

Использование Общероссийского математического портала Math-Net.Ru подразумевает, что вы прочитали и согласны с пользовательским соглашением http://www. mathnet.ru/rus/agreement

Параметры загрузки:

IP: 18.208 .226 .222

26 апреля 2023 г., 16:44:43

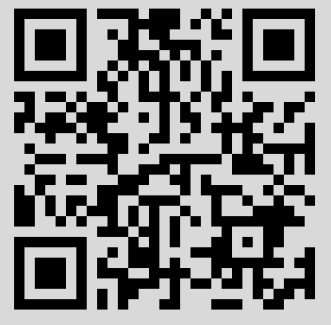




\section{ОЦЕНКА ПОГРЕШНОСТЕЙ АСТРОГИРОСИСТЕМЫ}

Рассматривается задача определения величины накопленной погрешности определения угла волоконно-оптическими гироскопами. Для космических аппаратов в качестве эталонной информации используются астродатчики. Измерения передаются на Землю для обработки, имеющей целью определение ориентации космического аппарата в инерииальной системе координат.

Введение. Волоконно-оптические гироскопы (ВОГ) хорошо известны как датчики угла или угловой скорости, принцип действия которых основан на эффекте Саньяка [1]. Они широко применяются в составе инерциальных бесплатформенных инерциальных навигационных систем (БИНС), устанавливаемых на морских судах, летательных аппаратах и других подвижных объектах [2]. Современные ВОГ имеют высокую точность в широком диапазоне измерения. Среди составляющих погрешности измерения ВОГ самый ощутимый вклад вносит уход гироскопа. Это связано с долговременным изменением сигнала, не связанным с движением исследуемого объекта. Факторы влияющие на величину ухода можно разделить на два типа, которые будем условно называть «определенные» и «неопределенные». К «определенным» относится, например, составляющая вектора угловой скорости Земли и связанное с ним постоянное смещение нулевого сигнала гироскопа, температурный дрейф и др. Эти величины известны и могут быть вычтены из исходного сигнала. К «неопределенным» относятся факторы для которых в настоящее время нет удовлетворительного математического описания и соответствующих количественных оценок. Целью данной работы является определение величины ухода гироскопа, вызываемой «неопределенными факторами.

Постановка задачи. Рассматривается задача оценки точности бесплатформенной инерциальной системы определения ориентации (БИСО) космического аппарата (КА) на основе ВОГ ООО «Оптолинк» с величиной ухода $0.05 \div 0.1$ угл. сек/сек. Предполагается, что на КА установлены астродатчики, позволяющие получать эталонную информацию по наблюдениям звезд.

Пусть на некотором интервале времени выполняются совместные измерения ВОГ и наблюдения звезд астродатчиками, в ходе которых определяется начальная ориентация КА и оцениваются параметры ухода ВОГ. Далее происходит интегрирование кинематических уравнений движения КА при известном начальном условии и компенсируется уход ВОГ. Для исключения влияния мультипликативных погрешностей БИСО, методы оценки которых приведены в [3], здесь рассматривается отдельный канал управления ориентацией КА.

Целью исследования является оценка точности БИСО в функции времени движения «в памяти», под которым понимается период, в течение которого ориентация определяется только на основе измерений ВОГ и оценок параметров его ухода. Для известных требований к точности измерений ориентации КА можно определить необходимую периодичность серий наблюдения звезд астросредствами, зависящую от предельной длительности движения «в памяти». Предварительно выполняются исследования выходного сигнала ВОГ методом вариаций Аллана [4] с целью определения границ диапазона вариации длительности движения «в памяти».

Метод оценки погрешностей астрогиросистемы. Задача решается методом статистического численного эксперимента, использующего записи сигналов ВОГ неподвижно устанавливаемых на испытательном стенде. В этом случае прибор измеряет интеграл от угловой скорости вращения Земли в проекции на свою ось чувствительности. Ввиду того, что скорость вращения Земли на интервале измерения можно считать строго постоянной, интеграл от угловой скорости представляет собой линейную функцию. Нулевой сигнал (вариации дрейфа) ВОГ в этом случае может быть получен как разность выходного сигнала прибора и его аппроксимации полиномом первого порядка, коэффициенты которого определяются по методу наименьших квадратов отклонений значений аппроксимационного полинома от измеренных значений угла. Типовой вид фрагмента нулевого сигнала приведен на рис. 1.

Для обеспечения независимости измерений весь интервал записи разбивается на последовательные фрагменты одинаковой длительности $T^{j} j=1, \ldots$, N. В свою очередь, каждый из фрагментов разбивается на 2 интервала: интервал аппроксимации $T_{a}^{j}$ и интервал движения «в памяти» $T_{p}^{j}$, таких что $T^{j}=T_{a}^{j}+T_{p}^{j}$. 
На каждом из фрагментов моделируется работа БИСО, а именно: на интервале периода ап-

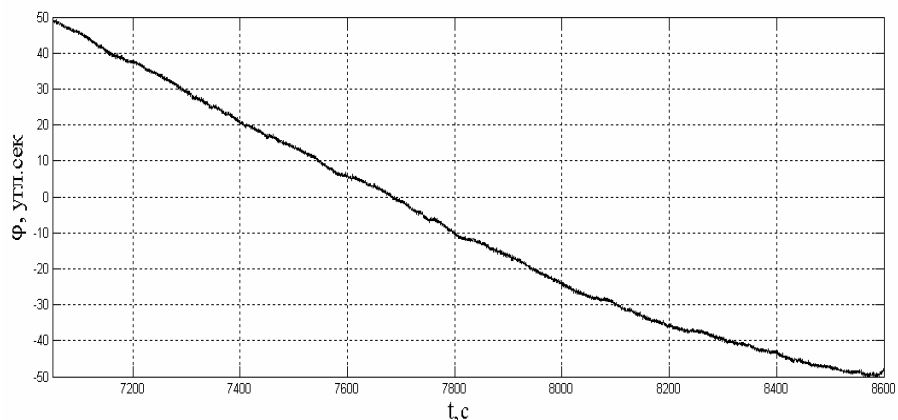

Р и с. 1. Типовой вид фрагмента нулевого сигнала проксимации $T_{a}^{j}$ (рис. 2) происходит выборка значений нулевого сигнала с периодом опроса астродатчиков; выбранная последовательность аппроксимируется полиномом первого порядка и на периоде работы «в памяти» $T_{p}^{j}$ определяются разности между значениями аппроксимирующей прямой и вариацией дрейфа ВОГ $\delta \varphi_{i}^{j}$; разности накапливаются в массивы с одинаковым индексом $i$ (т. е. для одинаковых моментов времени работы в памяти), но различными $j$ (т. е. для различных фрагментов сигнала на рис. 1) через равномерные промежутки времени $\Delta t$. Для каждого из $N \cdot T_{p} / \Delta t$ массивов вычисляется значения СКО вариаций дрейфа ВОГ.

Результаты численного эксперимента в виде графика зависимости СКО вариаций дрейфа

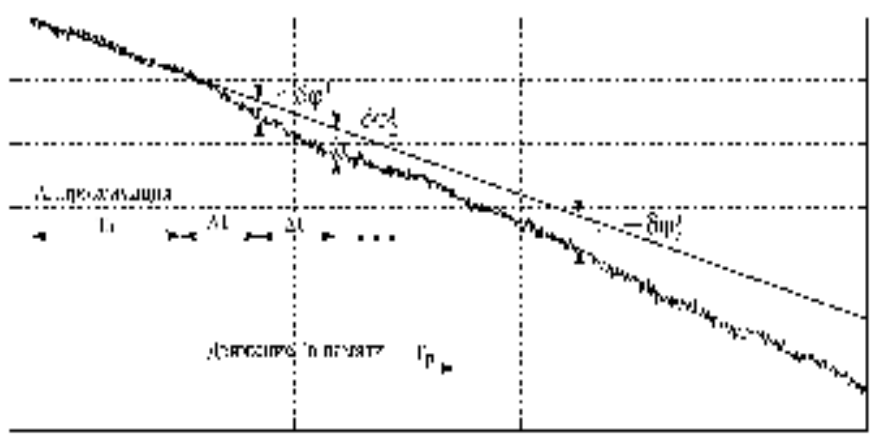

Р и с. 2. Определение участков аппроксимации и полета в «памяти» ВОГ в функции длительности работы в памяти для значений $T^{\mathrm{j}}=600 c, T_{a}^{j}=30$ сек, $j=1, \ldots, 90$, периода съема информации с астродатчиков 3 сек, $\Delta t=20$ сек приведены на рис. 3 , где номера $i-j$ означают: $i$ - номер прибора, $j$ - номер запуска.

Как показывает расчет, СКО погрешностей БИСО не превышает 10 " на периоде работы в «памяти» порядка 200 сек.

Представляет также интерес вариант постоянной коррекции аппроксимационного полинома с частотой измерения астродатчиков. Практическое применение этого варианта работы БИСО возможно в случае работоспособности астродатчиков при выполнении целевых задач КА, характеризуемых повышенными угловыми скоростями движения. Так как длительность работы в памяти фиксирована и равна периоду измерения астродатчиков, то варьируется длительность интервала аппроксимации Та. Результаты расчета при идеальном измерении астродатчиков (кривая 1) и при моделировании ошибки астродатчиков случайной величиной, распределенной по нормальному закону с $\sigma=2^{\prime \prime}$ и математическим ожиданием $m=1^{\prime \prime}$ (кривая 2) приведены на рис. 4

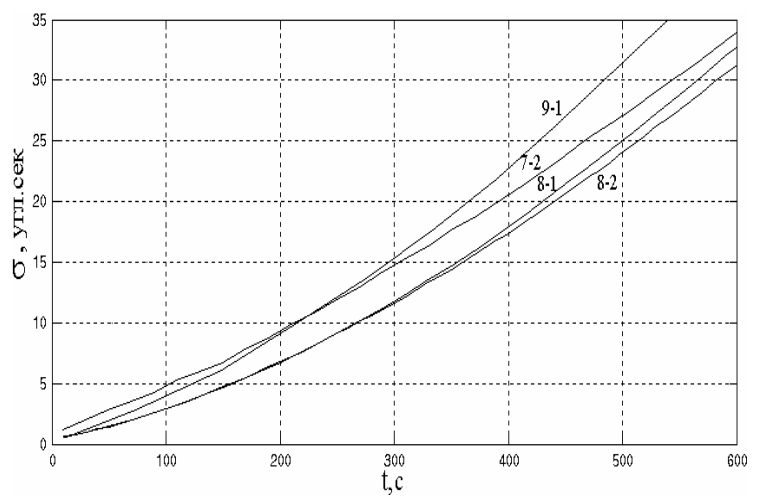

Р и с. 3. График зависимости СКО вариаций дрейфа ВОГ в функции длительности работы в памяти

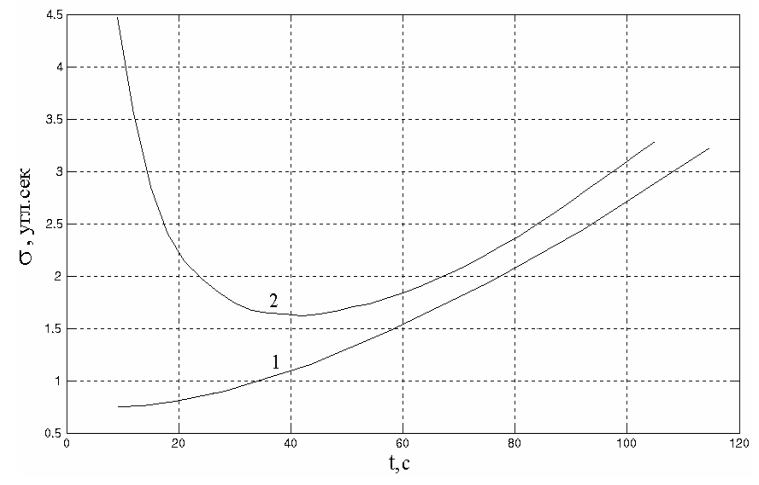

Р и с. 4. Результаты расчета:

1 - при идеальном измерении астродатчиков; 2 - при моделировании ошибки астродатчиков случайной величиной 
Как видно, оптимальная длительность периода аппроксимации $T_{a}=20-30$ сек. (7-10 измерений астродатчиков).

Заключение. Приведенная методика позволяет выполнить оценки достигаемой точности БИСО для заданной длительности полета в «памяти» по непосредственным измерениям нулевого сигнала ВОГ. Для варианта постоянной коррекции аппроксимационного полинома определена оптимальная длительность периода аппроксимации при заданных погрешностях наблюдения звезд астродатчиками.

\section{БИБЛИОГРАФИЧЕСКИЙ СПИСОК}

1. Lefevre H. The Fiber-Optic Gyroscope // Artech House, 1993. 280 p.

2. Анучин О. Н., Емельянцев $Г$. Н. Интегрированные системы ориентации и навигации для морских судов // М.: ГНЦ РФ ЦНИИ Электроприбор, 2003. 390 с.

3. Дюмин А. Ф, Егоров С. Н, Корабельщиков В. В, Суринский Д. М. Способ калибровки гироинерциальных измерителей бесплатформенной инерциальной навигационной системы ориентации космического аппарата. Патент РФ № 2092402, от 10.10 .97 г.

4. Кучерков С. Г., Лычев Д. И., Скалон А. И., Чертков Л. А. Использование вариации Аллана при исследовании характеристик микромеханического гироскопа //Гироскопия и навигация, 2003. № 2 (41). С. 98-103.

Поступила 13.09.2005 2. 